\title{
DEVELOPMENT OF MANILA HEMP FIBER EPOXY COMPOSITE WITH HIGH TENSILE PROPERTIES THROUGH HANDPICKING FIBER FRAGMENTS
}

\author{
KE LIU \\ School of Science, MOE Key Laboratory for Non-Equilibrium Synthesis and Modulation of Condensed Matter, \\ $X i$ 'an Jiaotong University, Xi'an, 710049, ShaanXi, People's Republic of China \\ Institute of Technology and Science, The University of Tokushima, 2-1 Minamijosanjima-cho, Tokushima \\ 770-8506 Japan \\ luecole@gmail.com \\ HITOSHI TAKAGI \\ Graduate School of Advanced Technology and Science, The University of Tokushima, 2-1 Minamijosanjima- \\ cho, Tokushima 770-8506 Japan \\ takagi@me.tokushima-u.ac.jp \\ ZHIMAO YANG
}

School of Science, MOE Key Laboratory for Non-Equilibrium Synthesis and Modulation of Condensed Matter, $X i$ 'an Jiaotong University, Xi'an, 710049, ShaanXi, People's Republic of China

zmyang@mail.xjtu.edu.cn

\begin{abstract}
Manila hemp fibers are separated to several sequent fragments from single fiber. The tensile strength of each fiber fragments and their epoxy composite are measured, followed by scanning electronic microscopic (SEM) analysis. The results show that the tensile strength of fiber fragments is almost constant along fiber. For composite, the tensile strength first increases and then decreases at the position near to root. The Young's modulus presents increasing with location from root to top for fiber and composite. Microstructure analysis indicates that the difference of tensile properties between fiber fragments derive from the difference of fiber diameter.
\end{abstract}

Keywords: Fiber fragments; composite; tensile properties.

\section{Introduction}

Within the past few years, there has been a dramatic increase in the use of natural fibers for composites. The potential of natural fiber-based composites using cellulose, wood, jute, kenaf, hemp, coir, sisal, pineapple, etc., as reinforcing fibers in both thermosetting and thermoplastic resins has received considerable attention among scientists all over the world for their advantages: (1) low density, (2) low cost, (3) low energy consumption, (4) high specific properties, (5) biodegradability, and so on ${ }^{1-3}$.

However, natural fibers show some disadvantages such as the lower mean value and large deviation on the mechanical properties. Natural fiber is essentially a composite in which rigid cellulose micro fibrils are helically embedded in a soft lignin and hemicellulose matrix ${ }^{4}$. Chemical composition (the content of cellulose) and cell structure 
of natural fibers (Spirally angle of fibrils and) are quite complicated. These parameters are greatly influenced by the climate condition, growth age, source and the digestion process, which in turn influence the properties of fiber and it's composite ${ }^{5,6}$.

It has been reported that fiber strength increases with increasing cellulose content and decreasing spiral angle in micro level ${ }^{5}$, and shows inverse dependence on diameter of fiber form macro view ${ }^{7,8}$. However, how growing point influences the properties of fiber has not been reported. Moreover, through simple method, how to obtain the fiber with high strength and thus enhance the mechanical properties of composite is unknown. In this paper, fiber fragments from different growing point in single Manila hemp fiber (MHF) and the corresponding epoxy composites are studied on mechanical properties. It is expected that the relationship between diameter, microstructure of Manila hemp fiber and the growing point can be known; in particular, based on fiber fragments, composite with high tensile properties can be developed.

\section{Experimental}

\subsection{Materials}

Manila hemp fiber employed in this work was obtained from Philippine with length no less than $1800 \mathrm{~cm}$. The epoxy resin was a product of Japan Epoxy Co., Ltd. (JER819, viscosity: 2 6, molecular weight: 180 200). Diethylene triamine (DETA) was used as curing agent.

\subsection{Preparation of fiber and it's composite}

Long Manila hemp fibers, which are selected without broken from root to top, were cut into series of sequential fiber fragments with about $12 \mathrm{~cm}$ long. Fiber fragments were rubbed by hands to removing the cuticle attached to the surface of fibers and then kept at $50^{\circ} \mathrm{C}$ for about $10 \mathrm{~h}$.

A resin formulation containing $7.0 \mathrm{wt} \%$ hardener was defoamed and mixed in vacuum mixer (Kurabo KK-250S), by which arranged fiber were casted directionally. Composites with size of $100 \mathrm{~mm} \times 10 \mathrm{~mm} \times 2 \mathrm{~mm}$ were obtained by pressing epoxy casting fiber in opensides alumina mold at $25^{\circ} \mathrm{C}$ for $24 \mathrm{~h}$, and then curing at $100^{\circ} \mathrm{C}$ for $8 \mathrm{~h}$. Composites with different fiber fragments $(30.0 \mathrm{wt} \%)$ are prepared under same pressure.

\subsection{Characterization}

In order to evaluate the tensile properties of fiber fragments and composites from different growing points. The fibers and composites were tested by electron universal materials testing machine (Instron 5567) with crosshead speed $0.5 \mathrm{~mm} / \mathrm{min}$, and $2 \mathrm{~mm} / \mathrm{min}$, respectively. For each fragments, 20 fibers are tested. To calculate the tensile strength and young's modulus, the cross-sectional area of each tested fiber are determined at three different points by laser video microscope.

The cross section of fibers was observed in scanning electronic microscopic (SEM). Prior to examination, the specimens were spurred coated with gold. 

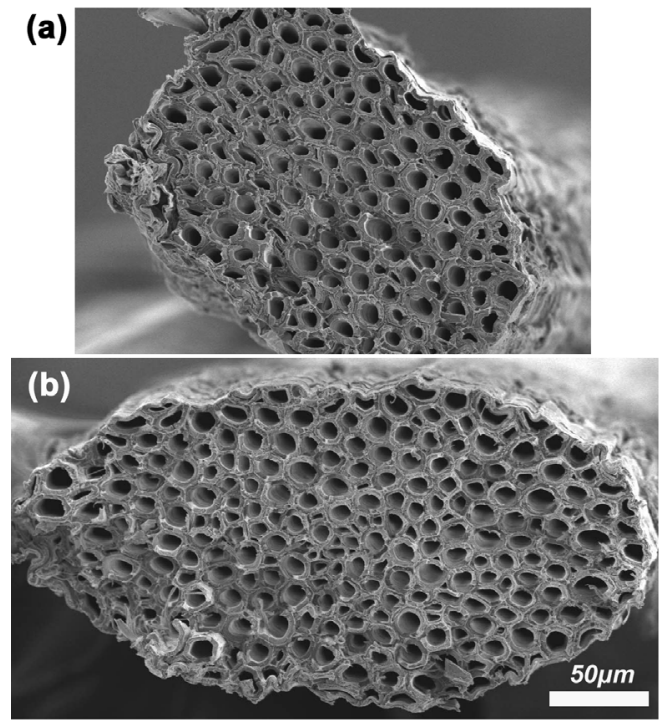

Fig. 1. Cross-sectional view of Manila hemp fiber fragments near (a) top and (b) root

\section{Results and discussion}

\subsection{Variation among Manila hemp fiber fragments}

Fiber fragments from different growing points are marked by the distance from root (DR) of MHF. The average cross-sectional area and load capacity of each fiber fragments are plotted in Fig. 2. It can be seen that the cross-sectional area of fragments decrease with DRT increasing. It agrees with the common sense. Fig. 1 (a) and (b) are the typical cross section view of fiber fragments at about $160 \mathrm{~cm}$ and $20 \mathrm{~cm}$ from fiber root, respectively. From this figure, it can be seen that fiber fragments near to top is thinner than that near to root. Fig. 2(b) shows that the load capacity of fiber fragments decrease with distance increase except the first fragment, which may results from the extracting damage when the long fibers are treated by raw materials supplier.

Fig. 3 presents that the relation between tensile properties of fiber fragments and distance. We can see that tensile strength is almost constant with growing point moving to fiber top shown in Fig. 3(a) except the first fragment. The reason why different fiber diameter leads to the same tensile strength may results from the deformation of fiber bundle during the tensile test, which derives from the uncoiling of spirally oriented fibrils ${ }^{4}$. The detail failure mode needs further analysis, which cannot be described simply in this paper.

Fig. 3(b) shows that Young's modulus increase greatly as the distance increasing. Correspondingly, the strain of fiber shows decreasing with growing point moving from root to top (see Fig. 5(a)). It is easy to see that the number of microfibers in fiber fragments near top is larger than the one near root as shown in Fig. 1, therefore, for fiber fragments near top, it is easier for stress to transfer along fiber bundle under smaller 
deformation of microfiber in the direction across fiber, i.e. smaller strain of fiber bundle in fiber direction, which leads the increasing of Young's modulus and decreasing of strain.

(a)

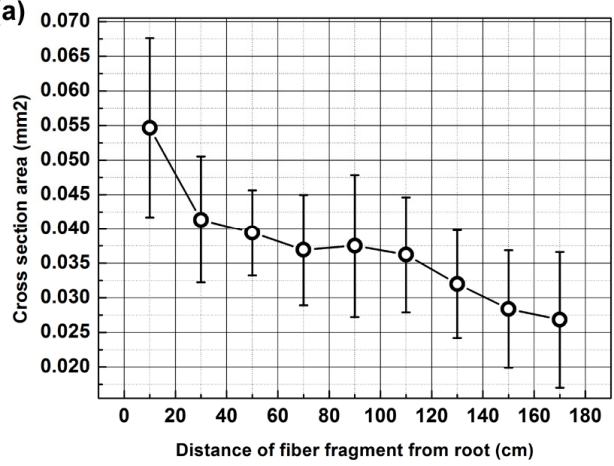

(b) 26

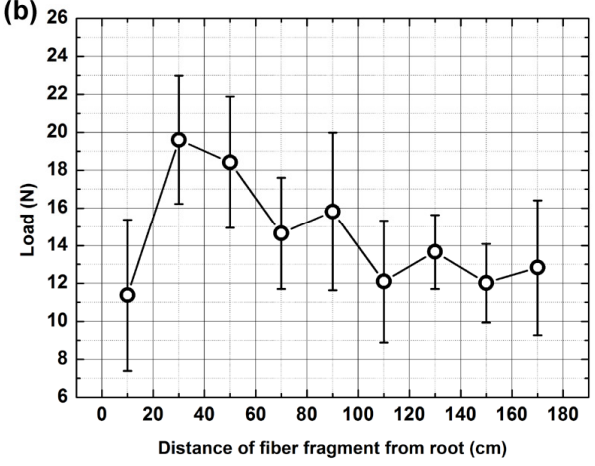

Fig. 2. (a) Cross section area and (b) load capacity of MHF fragments vary with distance of fiber fragments from root
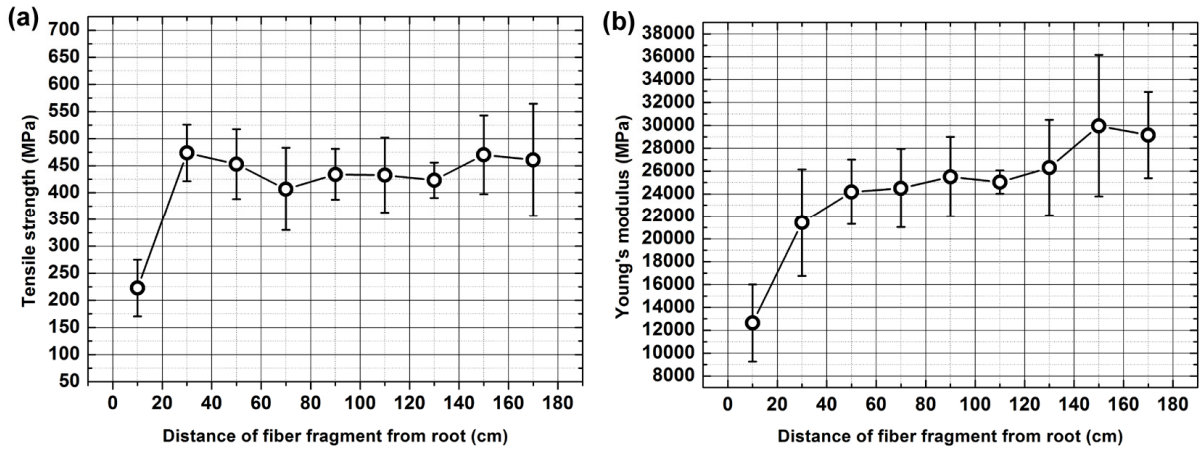

Fig. 3. (a) Tensile strength and (b) Young's modulus of MHF fragments vary with distance of fiber fragments from root

\subsection{Variation among composites with MHF fragments}

Different to MHF fragments, the tensile strength of composites decreasing with DR increasing as shown in Fig. 4(a). Because surface of fiber is untreated, interface between fiber and resin becomes the weak region in composite. Diameter of fiber fragments near top (FFT) is smaller than that if fiber fragments near root (FFR), thus, content of interface in composite with FFT is higher than the one with FFR. As the applied stress reaches the tensile strength of microfibers, they will be ruptured. Therefore, FFT in composite is broken at the same stress with FFR in composite. For natural fiber composite, there is the second step of composites damage: the crack propagates along the weak interface 9 Therefore, it is easier for crack to extend the whole materials in composite of FFT with more interfaces, i.e. the tensile strength of composite decreases with DR increasing. Furthermore, the more brittle fiber fragments near top (see Fig. 5(a)) maybe the other reason for the decreasing tensile strength of composite with DR increasing, which can be 
demonstrated from the strain tendency of composite (Fig. 5(b)). Based on the fracture mode of natural fiber composite, it is of high possibility to fabricate unidirectional MHF composite with high tensile strength through surface modification, especially for using fiber fragments near top.
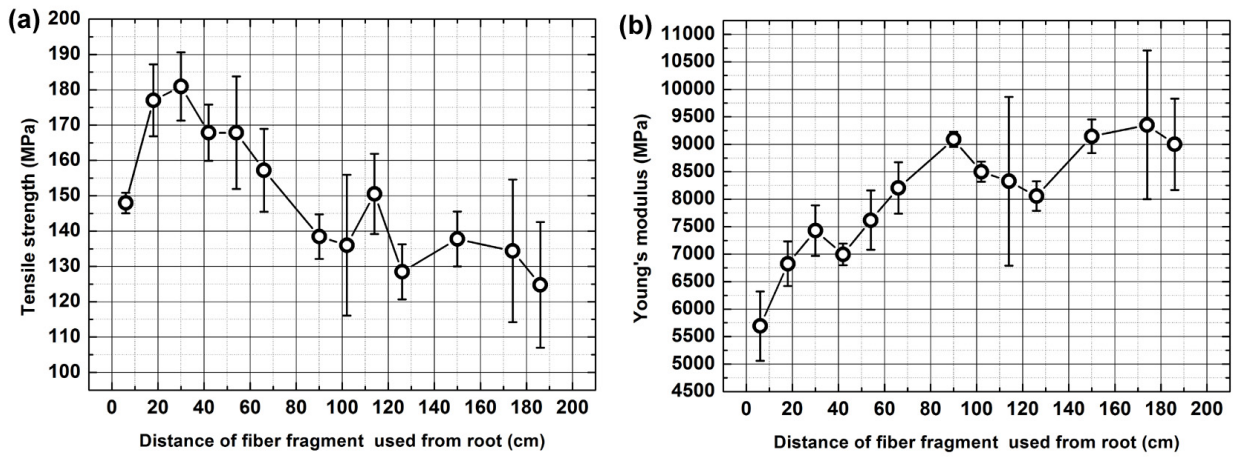

Fig. 4. (a) Tensile strength and (b) Young's modulus of composites with MHF fragments vary with distance of fiber fragments from root

From Fig. 4(b), it can be seen that Young's modulus of composites increasing with DR increasing. Before fiber is ruptured, stress transfer in fiber and resin based on the rule of mixture, therefore, Young's modulus of composite presents the same tendency with that of fiber fragments as shown in Fig. 3(b).
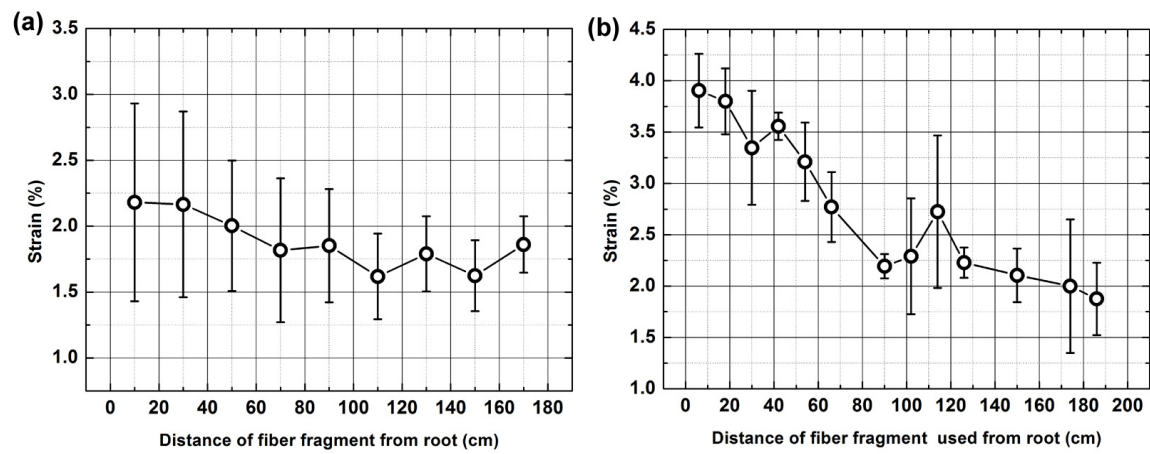

Fig. 5. Strain of (a) MHF fragments and (b) composites with MHF fragments vary with distance of fiber fragments from root

Therefore, if we need a composite with high tensile strength, fiber fragments near root should be used, if composite with high Young's modulus is desired, we should utilize fiber fragments near top. Surface modification is an approach to obtain composite with high strength and modulus, where FFT is used. 


\section{Conclusions}

Sequent fragments of Manila hemp fiber and their epoxy composites have been prepared, of which the tensile properties was studied. From different growing point, tensile strength of fiber fragment shows constant value, while fiber fragments near top present high Young's modulus. It derives from the diameter decreasing with growing point moving to top. For composite, tensile strength decrease and Young's modulus increases with distance from root increasing. This is different to the case of fiber due to the weak fiber resin interface and brittle fiber near top. Composite with high strength and modulus can be obtained through interface modification and using fiber near top.

\section{Acknowledgments}

This work was partially supported by the presidential research grant of The University of Tokushima and Double-Degree Exchanged Program between The University of Tokushima (Japan) and Xi'an Jiaotong University (China).

\section{References}

1. A. Mohanty, M. Misra, and G. Hirichsen, Macromol Mater Eng, 276, 1-24 (2000).

2. A. Netravali, Advanced Composite materials, 16, 269-282 (2007).

3. D. N. Saheb and J. P. Jog, Advances in Polymer Technology, 18, 351-363 (1999).

4. M. Z. Ronga, M. Q. Zhang, Y. Liu, Composites Science and Technology, 61, 1437-1447 (2001).

5. A. K. Bledzki and J. Gassan, Progress in Polymer Science, 24, 221-274 (1999).

6. S. Mishra, A. K. Mohanty, L. T. Drzal, Macromolecular Materials and Engineering, 289, 955-974 (2004).

7. A. B. Bevitori, I. L. A. D. Silva, F. P. D. Lopes, Revista Matéria, 15, 125-131 (2010).

8. W. P. Inacio, F. P. D. Lopes, and S. N. Monteiro, Revista Matéria, 15, 176-182 (2010).

9. S. L. Bai, R. K. Y. Li, L. C. M. Wu, Journal of materials science letters, 17, 1805-1807 (1998). 